\author{
Proceedings of the \\ $1^{\text {st }}$ International Conference and Exhibition on Future RFID Technologies \\ Eszterhazy Karoly University of Applied Sciences and \\ Bay Zoltán Nonprofit Ltd. for Applied Research \\ Eger, Hungary, November 5-7, 2014. pp. 127-134 \\ doi: 10.17048/FutureRFID.1.2014.127
}

\title{
Software system for processing and analysis of fingerprints and determination of necessary parameters
}

\author{
Pavol Marák, Alexander Hambalík \\ Institute of Computer Science and Mathematics \\ Slovak University of Technology in Bratislava, Slovakia \\ pavol.marak@stuba.sk, alexander.hambalik@stuba.sk
}

\begin{abstract}
The aim of this contribution is to discuss automated fingerprint feature extraction and its inherent parameters that influence the performance of extraction. This paper primarily deals with our fingerprint processing and analysis software from the perspective of used parameters and their determination. Our system consists of two consecutive stages: image preprocessing and minutiae extraction. We discuss the specific parameters in the order given by the software architecture. All the parameter settings are based on the native fingerprint image resolution to obtain optimal results. In case of image preprocessing methods we discuss parameters of fingerprint pattern segmentation and image enhancement by using Gabor filter. It is followed by minutiae extraction stage that involves two algorithms based on different principle. First, it is minutiae extraction based on ridge skeleton analysis and the other mentioned is minutiae extraction using ridge contours. Given the first algorithm, we consider these crucial parameters: minutiae layout shift before and after transforming fingerprint into skeleton and determination of distance between consecutive pores along the ridge. In the second algorithm, the most important aspects to consider are length specification of analyzed ridge segment and computation of ridge curvature. This paper presents results of our research conducted on a sample of 1140 fingerprint images, provided by the Institute of Forensic Science in Bratislava, Slovakia.

Keywords: fingerprints, minutiae, biometrics, extraction, features, pattern recognition
\end{abstract}




\section{Introduction}

Biometrics in its various forms has become an inherent part of our everyday life. With the growing popularity of embedded systems and smart mobile devices we face the question of data security and verification of identity to keep all our data safe and accessed only by authorized people. Fingerprints are one of the many known biometric traits with the unmatched advantages that make them biometric technology number one at the moment. However, fingerprint recognition is not a completely solved problem. We frequently see many new algorithms promising improved performance on poor quality fingerprints. It is more or less difficult to say which recognition algorithms or entire complex systems are generally the best or most suitable.

It depends on the application scenario rather than computing power or misleading results of testing on some narrow or very specific database of fingeprints. Traditionally, it is believed that popularity of fingerprints in biometric community can be attributed to their historical origins and scientific works written by Francis Galton (1892) or William Herschel (1916) who were among the first to discover the uniqueness and permanence of fingerprints and lay the groundwork for their use in criminal investigation. Today, automated biometric systems as we know them, are still based on extraction and matching special characteristic patterns, where the most valuable are so called minutiae points.

Well-balanced and reliable fingeprint recognition algorithm always depends on feature set it uses for matching. Fingerprints possess various patterns starting with global ridge flow and ending with very fine details on microscopic level. Minutiae points are generally the most widely used feature because of their relative immutability in fingerprint pattern thus ensuring stable representation of single fingerprint even under variable sensing conditions. Another important aspect of fingerprint recognition is its evaluation using a suitable database of fingerprints of all possible global patterns and varying quality.

Minutiae extraction is very crucial problem and it needs to be adressed. There are more minutiae types, but automated systems are taking into account mainly bifurcations and endpoints. Therefore a lot of rare and expressive information is lost. In this work we want to emphasize the role of fingeprint extraction, we want to outline software implementation of minutiae extraction and demonstrate the impact of the chosen parameters on the success of the whole system. As an example we describe our fingeprint feature extraction algorithm that is able to recognize more minutiae types.

\section{Research goals}

There is a lack of serious software fingerprint analysis solutions on a larger scale (population-wide, country-wide or even within the entire continent). Such an analysis, carried out manually by professional forensic examiners would be a tedious process without the use of computer power and suitable software equipment. To de- 
velop a software solution capable of complex fingerprint analysis was the primary goal of our research. Mainly we wanted to deal with open issues of fingerprint processing. Specific goals are as follows:

- Design and development of software solution for automated fingerprint processing

- fingerprint preprocessing

- minutiae extraction (extending previous approaches with ability to extract more complex patterns)

\section{- Specification of neccesary parameters}

- explaining effect of important parameters on overall performance

\subsection{Motivation}

It is important to explain key factors of our motivation of our research by answering these questions:

- What do we want to achieve?

- develop a new method of fingerprint feature extraction

- incorporate complex features that have not been adopted yet

- make forensic examiner experience a part of our know-how

- Why is it important to deal with this problem?

- improving performance of fingerprint recognition

- improving performance on low quality images

- statistical analysis of fingerprint features for better understanding their distribution

- Where can we make use of it?

- field of forensic science

- logical and physical access control

Typical operations of fingerprint recognition system are depicted by diagram in Figure 1. Our research focuses directly on the stage of image preprocessing and feature extraction. 


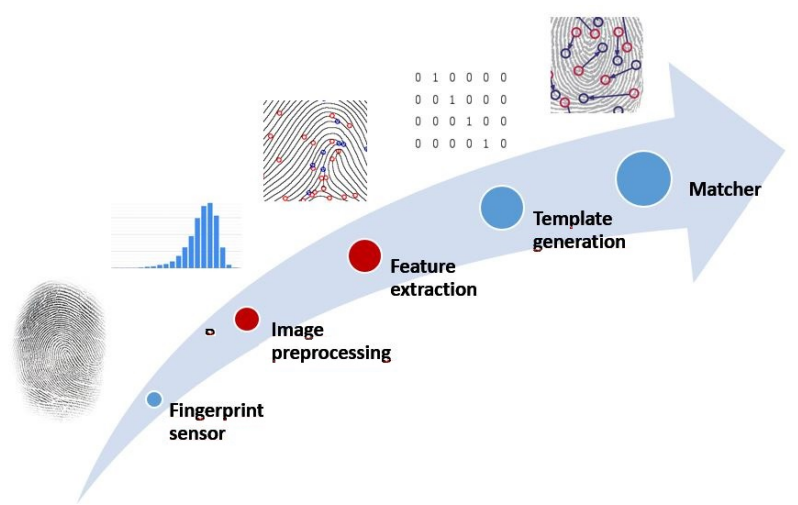

Figure 1: Stages of automated fingerprint processing

\section{Fingerprint features}

In general there are 3 levels of fingerprint features. Level-1 features are visible ridge flow patterns that are used for rough classification purposes only. Overall ridge flow normally fall within one the categories, arc, loop and whorl.

Level-2 features are local ridge characteristics sometimes called minutiae as we mentioned before. In Figure 2 we can see classification scheme of minutiae provided by Institute of Forensic Science in Bratislava, Slovakia. They are associated with their relative frequency they occur in Slovak population represented by 1000 sample fingerprint images.

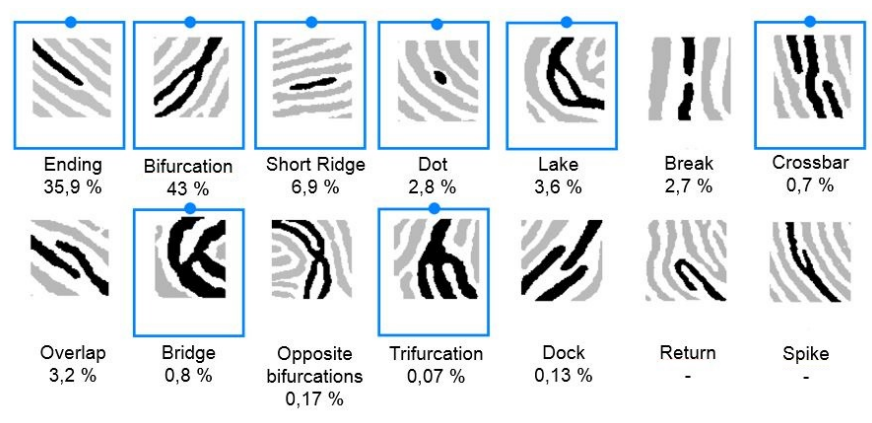

Figure 2: Level-2 fingerprint features - minutiae

Current biometric systems mostly rely on two minutiae types: ridge bifurcations and terminations. Ability to distinguish more than two types of fingerprint features is of high importance. Patterns extracted by our software are highlighted by blue color: ending, bifurcation, short ridge, dot, lake, crossbar, bridge and trifurcation minutia respectively. What can be viewed as an innovative feature in our software 
is its ability to extract up to 8 minutiae shape types, it is $94 \%$ of known features while other existing solutions are usually based only on first two types, that is $79 \%$. But we need to bear in mind, that the lower the frequency in population, the higher value for identification it provides.

At the very-fine level, small details, Level-3 features can be detected. These include ridge edges, pores, scars, etc. Their extraction is feasible only in high resolution of $1000 \mathrm{ppi}$ or more. More and more research is dedicated to their research in automated biometric systems as high resolution sensors become available.

\section{Software solution and important parameters}

Figure 3 describes architecture of our fingerprint processing solution. It is divided into two consecutive modules: image preprocessing and minutiae extraction.

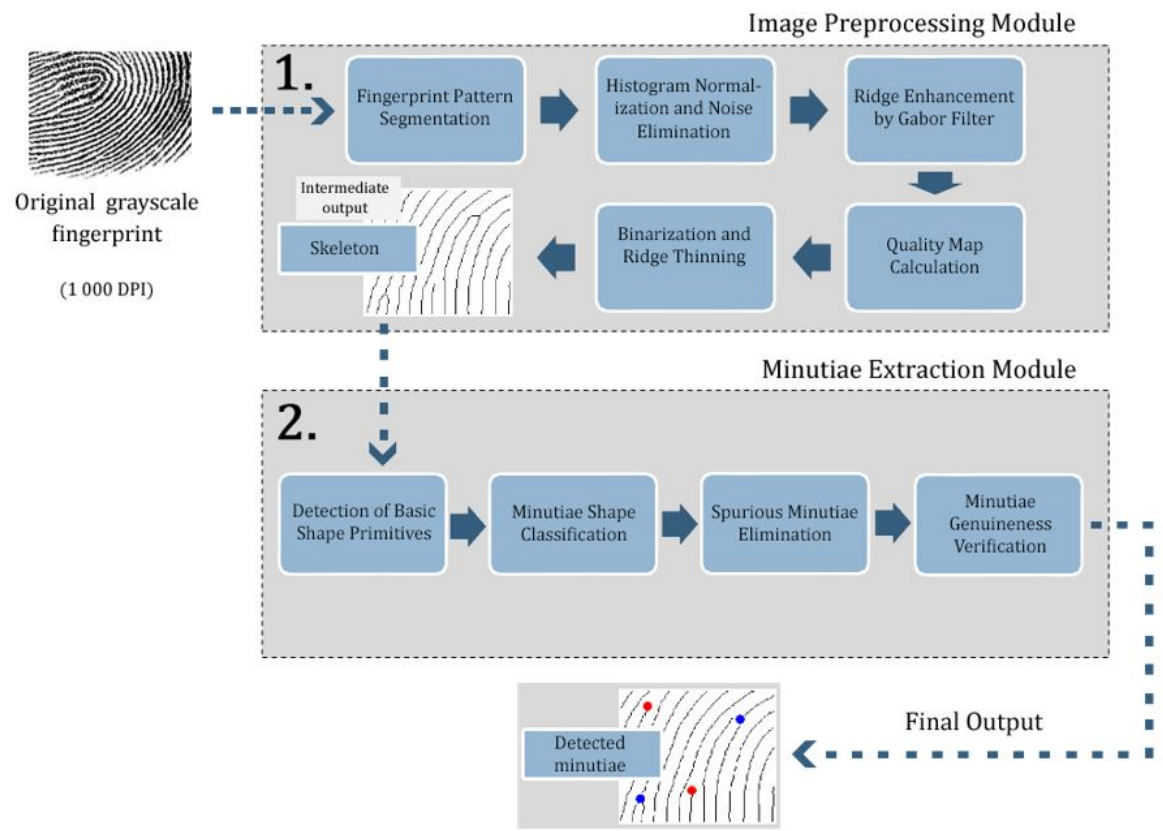

Figure 3: Architecture of proposed fingerprint analysis system

The original grayscale fingerprint image is first passed to image preprocessing module, where it is transformed by a series of enhancement techniques into the appropriate form for minutiae extraction. This form is fingerprint skeleton. In the extraction module, minutiae are detected in skeleton image using specific topological rules. Finally, false minutiae are filtered out. At the minutiae classification 
stage, we use extended topological analysis or ridge contour analysis. The final product is a set of located minutiae in fingerprint image along with their direction, quality and additional very important characteristics like ridge direction map and ridge frequency map.

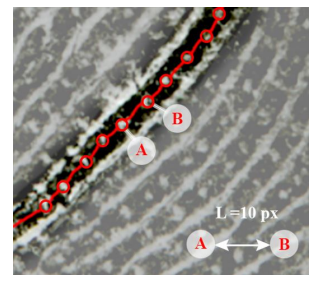

Figure 4: Building blocks of ridge lines

Before we start explaining our techniques, let us explain one fingerprint attribute that we used to derive algorithm parameters. To be able to analyze fingerprint, we need to respect its structure that is given by the ridges. Ridges are composed of small blocks, the length of particular block is distance between 2 consecutive pores as can be seen in Figure 4, letters A and B correspond to consecutive pores along the ridge. This length is denoted as $\mathrm{L}$ and we found that the average value is 10 pixels at resolution of $1000 \mathrm{ppi}$. This value is used to extract minutia during extended topological analysis. All minuatia type dimensions and their components can be expressed in terms of $\mathrm{L}$.

\subsection{Image preprocessing}

In this section we discuss chosen parts of preprocessing scheme from Figure 3. Fingerprint segmentation, orientation map computation and Gabor filtering is presented. We presume that these steps are suitable for demonstration of what effect might parameter change have on outputs of these algorithms.

Segmentation Here, the goal is to separate fingerprint pattern from unnecessary background pixels. The idea behind this technique is based on fact that fingerprint pattern is characterized by alternating darker and brighter stripes, this causes a high variance of color intensity. We divided the image into the blocks and computed the variance in each and compared them to a treshold to discard those blocks with low variance. This way we obtained fingerprint image separated from background, so called region of interest. The key parameter was block size for variance computation. Optimum results were achieved with size set to $\mathrm{L} / 2$ because in average it contained 1 ridge-valley structure that was quite enough to capture characteristic gray-scale variance. Bigger sizes resulted in inaccurate border detection.

Image enhancement by applying Gabor filters Enhancement of ridge clarity needs application of so called context filters. We chose a Gabor filter. We assume 
that fingerprint can be viewed as a composition of sinusoidal planes. Gabor filter is a function that can be adjusted to model the fingerprint structure, it is a sine function modulated by Gaussian function. It reconstructs the indistinct structure of fingerprint. To be able to perform filtering we need to compute the direction map and frequency map of fingerprint to adjust the filter. Then, we can apply the filter.

- Filter direction

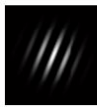

- Filter frequency

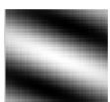

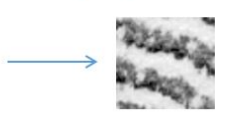

- $\quad$ Filter aspect ratio
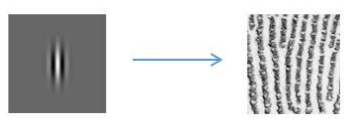

Figure 5: Gabor filter parameters and their appropriate setting in different fingerprint regions

There are 3 key parameters of Gabor filter: direction, frequency and aspect ratio. By their accurate estimation we can fine tune the filter to match the local fingerprint pattern. Figure 5 shows filter parameters as should be set in order obtain optimum enhancement for particular image counterparts. Here are the recommendations of their optimum values:

- filter direction is expected to be similar to local ridge direction

- filter frequency is expected to be similar to the frequency of ridges in small image block

- aspect ratio defines ellipticity of the filter, elliptic filter shape is good choice when working with image parts that do not have significant direction change and square filter is used when direction changes rapidly.

\subsection{Minutiae extraction}

We proposed two methods of minutiae extraction based on different input fingerprint representations. While extended topological examination works with skeleton images with ridge thickness of 1 pixel, contour analysis is processing the original binarized fingerprint leaving all shapes almost untouched.
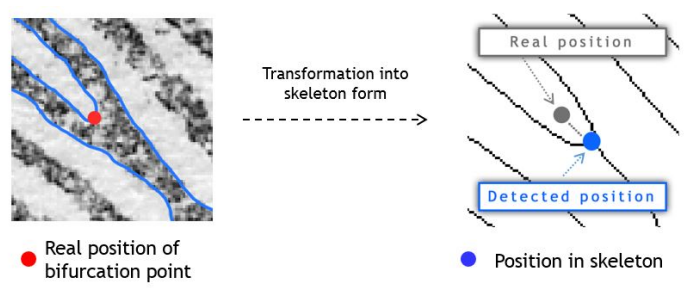

- Position in skeleton

Figure 6: A problem of minutiae position shift in the skeleton image 
Using skeleton image as input introducess some problems with coordinates of minutiae. To assess the accuracy of extraction of various minutiae types, we need to compare minutiae extracted programatically and manually. Before we can do so, we need to shift minutiae coordinates in skeleton to such an extent so they correspond to original ridge lines in original fingerprint. We need to fix the incorrect position by shifting coordinates by $\mathrm{L} / 2$ pixels in direction of the ridge. On average, this value represents half the width of the ridge line.

The countour analysis uses ridge line contours and measures the curvature. Minutia point is detected if the curvature exceeds some threshold. In this algorithm, the most sensitive parameter is length of the segment of the ridge to be measured. Small values are not able to capture minutiae shape and are prone to noise. Higher values on the contrary might cause confusion by detecting more minutiae in one ridge line. It is clear that it is necessary to determine a trade-off.

\section{Conclusion}

In this paper we discussed automated feature extraction from the perspective of important parameters that determine overall algorithm outcome. We demonstrated our own fingerprint analysis solution as a chain of multiple processes restoring the pattern structure and extracting characteristic data. A special emphasis was put on visualization of link between fingerprint features and critical parameters.

Acknowledgements. This research is supported by:

- Operational Program of Research and Development: project "Centrum excelentnosti bezpecnostneho vyskumu"(ITMS code 26240120034)

- VEGA 1/0173/13 project "Privacy on mobile devices"

- Slovak University of Technology, Programme for supporting young scientists: Pokracujuce projekty na podporu excelentnych mladych vyskumnikov, Project acronym: "FingerDetective2014"

- "Secure implementation of post-quantum cryptography"- SPS Project Number: 984520, NATO Science for Peace and Security Programme

\section{References}

[1] Maltoni, D., Et AL., Handbook of Fingerprint Recognition: Second Edition, Springer (2009), 494 p., ISBN 978-1-84882-254-2.

[2] Fahmy, M. F., Thabet, M. A., A novel scheme for fingerprint enhancement, 31st National Radio Science Conference, (2014), p. 142 - 149., ISBN 978-1-4799-3820-9. 